\title{
PALHA DE TREVO VESICULOSO (Trifolium vesiculosum Savi cv. Yuchi) COMO SUBSTITUTO DA SILAGEM DE MILHO NA ALIMENTAÇÃO DE NOVILHOS CONFINADOS ${ }^{1}$
}

\author{
CLOVER STRAW (Trifolium vesiculosum Savi c.v. Yuchi) AS A SUBSTITUTE \\ OF CORN SILAGE IN THE FEEDING OF CONFINED STEERS
}

\author{
João Restle ${ }^{2}$ Estevão Pascoalini Dias Nucci ${ }^{3}$ Jorge Luis Carvalho Flores ${ }^{4}$
}

\section{RESUMO}

\begin{abstract}
O experimento teve como objetivo avaliar a utilização da palha de trevo vesiculoso como substituto parcial da silagem de milho, na fração volumosa da dieta fornecida para novilhos confinados, sendo testados os seguintes níveis de substituição: T0 constituído por 100\% de silagem de milho; T25 constituído por $75 \%$ de silagem de milho mais $25 \%$ de palha de trevo vesiculoso; 750 por $50 \%$ de silagem de milho mais $50 \%$ de palha de trevo vesiculoso e T75 constituído por $25 \%$ de silagem de milho mais $75 \%$ de palha de trevo vesiculoso. O teor de concentrado na dieta oferecida foi de $32,5 \%$. As dietas foram fornecidas durante 112 dias para novilhos com peso e idade inicial de $265 \pm 1,65 \mathrm{~kg}$ e 18 meses, respectivamente. Os dados foram submetidos à análise de regressão para nível de substituição (NS) de silagem de milho por palha de trevo vesiculoso na fração volumosa da dieta. O consumo voluntário de matéria seca (CMS em $\mathrm{kg}$ de MS/100kg PV (CMSP) ou em g/kg PV $V^{0,75}$ (CMSUTM) decresce linearmente à medida que aumentou o nível de palha de trevo. O consumo de energia digestivel diário expresso em Mcal/100kg PV (CEDPV) também decresceu linearmente conforme a equação: $C E D P V=7,22905-0,025941 \mathrm{NS}$. O ganho de peso médio diário (GMD) observado foi de 1,$25 ; 1,18 ; 1,07 e$ 1,01 , respectivamente. A análise de regressão mostrou que o aumento de uma unidade percentual de substituição de silagem de milho por palha de trevo provocou um decréscimo de $3,47 \mathrm{~g}$ no GMD e piorou a conversão alimentar em 0,017 unidades. Apesar de causar uma redução no desempenho animal, a inclusão de palha de trevo vesiculoso na fração volumosa da dieta de bovinos é viável, tendo em vista que, mesmo no nível de substituição mais elevado, o GMD dos animais foi de um quilograma, o que demonstra o seu potencial de utilização na produção de bovinos.
\end{abstract}

Palavras-chave: Charolês, consumo voluntário, eficiência alimentar, ganho de peso, palhada, trevo vesiculoso, resíduo de cultura.

\begin{abstract}
SUMMARY
The objective of the experiment was to evaluate the use of clover straw as partial substitute for corn silage in the roughage fraction of the diet of confined steers, being tested the following substitution levels: T0 with 100\% of corn silage; T25 with $75 \%$ corn silage and $25 \%$ clover straw; T50 with $50 \%$ corn silage and 50\% clover straw; T75 with $25 \%$ corn silage and $75 \%$ clover straw. The concentrate content in the diet was $32.5 \%$. The diets were fed during 112 days for steers with an initial average weight and age of $265 \pm 1.65 \mathrm{~kg}$ and 18 months, respectively. The data were submitted to regression analysis, for the substitution level (SL) of corn silage by clover straw in the roughage fraction of the diet. Dry matter intake (DMI) expressed in $\mathrm{kg}$ of DM/100 kg of LW (DMIP) or in $\mathrm{g} / \mathrm{kg} P V^{75}$ (DMIM) declined linearly as the level of clover straw increased. Daily digestible energy intake expressed in Mcal/100 $\mathrm{kg}$ of LW (DEIP) also declined acoording to the following regression equation: DEIP $=7.22905-.0259$ $(S L)$. The observed average daily weight gain $(A D G)$ was 1.25; $1.18 ; 1.07$ and $1.01 \mathrm{~kg}$, respectively. The regression analysis showed that the increase of one percentile unit of substitution of corn silage by clover straw, caused a decline of $3.47 \mathrm{~g}$ on the $A D G$ and worsen the food convertion in .017 units. Although causing a reduction in animal performace, the inclusion of clower straw in the diet of cattle is viable, considering that even in the highest level of substitution the ADG of the steers was one kilogram.
\end{abstract}

\footnotetext{
${ }^{1}$ Parte da Dissertação de Mestrado, apresentado pelo segundo autor ao Curso de Pós-graduação em Zootecnia, UFSM, para obtenção do grau de Mestre em Zootecnia.

2 Engenheiro Agrônomo, PhD., Professor Titular, Departamento de Zootecnia, Universidade Federal de Santa Maria (UFSM), Pesquisador do CNPq, CEP 97105-900, Santa Maria, RS. E-mail: restle@ infoway.com.br. Autor para correspondência.

${ }^{3}$ Médico Veterinário, MSc.

${ }^{4}$ Zootecnista, MSc.
} 
Key words: Charolais, voluntary intake, food convertion, weight gain, straw, clover vesiculosum, crop residue.

\section{INTRODUÇÃO}

O trevo vesiculoso é uma leguminosa anual de estação fria utilizada como forrageira na região sul do país. A utilização dessa leguminosa, geralmente associada a gramíneas anuais de inverno, tem resultado em elevado ganho de peso médio diário por animal e por ha (QUADROS \& MARASCHIN, 1987; SILVA et al., 1997). Além de sua utilização como forrageira, a produção de semente de trevo vesiculoso é um componente importante na rentabilidade do sistema, tendo em visto o elevado valor comercial que essa atinge no mercado. Além disso, após a colheita de sementes, a palha pode ser enfardada e armazenada para posterior alimentação de ruminantes.

As palhas produzidas após a colheita de grãos ou sementes, geralmente, estão desidratadas, podendo ser colhidas e armazenadas imediatamente após o processo de trilhagem, o que faz com que o risco de produção seja pequeno. Além disso, o seu custo de produção é baixo quando comparado com outras formas de confecção e armazenagem de volumosos. A utilização de palhas produzidas pelas lavouras para a alimentação animal é pequena, embora o Brasil apresente um grande potencial agrícola. Nos EUA, as palhas participam com 25 a $50 \%$ das rações fornecidas aos animais nos meses de inverno, sendo utilizadas tanto de forma exclusiva, como misturadas a concentrados, ou consumidas diretamente pelos animais no campo (ANDERSON, 1978). A utilização exclusiva de palhas na alimentação de bovinos resulta em perdas de peso, como verificaram BAGLEY et al. (1989), utilizando palha de soja na alimentação de novilhos. No entanto, essas perdas são inferiores às perdas verificadas nos animais mantidos exclusivamente em campo nativo durante o inverno (MÜLLER et al., 1979). Essa perda de peso está associada à qualidade das palhas, que apresentam baixo valor nutritivo, com teores de proteína e energia baixos. Além disso, possuem altos teores de fibra devido aos níveis de carboidratos estruturais de altas frações indigestíveis (CRUZ, 1992; QUEIROZ et al., 1998; RESTLE et al., 2000). Essas características das palhas contribuem para a redução do consumo total de matéria seca, conseqüentemente, ocorre uma menor ingestão de nutrientes necessários para atender às necessidades energéticas para mantença e produção dos animais.

A utilização de palha associada a concentrado na alimentação de bovinos tem mostrado bons resultados. RESTLE et al. (1992), utilizando na alimentação de terneiros confinados $30 \%$ de concentrado associado a $70 \%$ de palha de trevo vesiculoso, feno de milheto ou feno de sorgo forrageiro, obtiveram ganhos de peso médio diário de 505, 508 e $522 \mathrm{~g}$, respectivamente. As palhas também podem ser usadas na alimentação de bovinos associadas a outros volumosos de melhor qualidade, com bons resultados. No entanto, mesmo quando associadas a esses volumosos, as palhas provocam uma redução no consumo de matéria seca e no ganho de peso dos animais, como verificaram RESTLE et al. (2000), utilizando palha de soja em substituição à silagem de sorgo forrageiro na alimentação de terneiros confinados. No entanto, os autores concluem que, mesmo no nível mais elevado de substituição, a palha de soja proporciona ganho de peso satisfatório aos animais.

A utilização de forrageiras como o trevo vesiculoso para pastejo direto, com subseqüente produção de sementes e utilização da palha produzida, são importantes no sistema de produção de bovinos de corte, em que se busca cada vez mais a eficiência como um todo. São praticamente inexistentes na literatura informações sobre a utilização de palha de trevo vesiculoso na alimentação de bovinos. Portanto, este experimento tem como objetivo avaliar o potencial da palha de trevo vesiculoso como substituto parcial da silagem de milho na alimentação de novilhos, através do seu desempenho em confinamento.

\section{MATERIAL E MÉTODOS}

O experimento foi conduzido, no Setor de Gado de Corte do Departamento de Zootecnia da Universidade Federal de Santa Maria - UFSM. Foram testados os seguintes níveis de substituição (NS) da silagem de milho por palha de trevo vesiculoso na fração volumosa da dieta: T0 constituído por $100 \%$ de silagem de milho; T25 constituído por $75 \%$ de silagem de milho, mais $25 \%$ de palha de trevo vesiculoso; T50 constituído por $50 \%$ de silagem de milho, mais $50 \%$ de palha de trevo vesiculoso e T75 constituído por $25 \%$ de silagem de milho, mais $75 \%$ de palha de trevo vesiculoso.

A palha de trevo vesiculoso foi produzida em uma área que foi utilizada com animais em pastejo de 15 de maio a 15 de outubro, quando foram retirados os animais, sendo a semente colhida em 18 de janeiro. Logo após a colheita das sementes com uma automotriz, a palha foi enfardada através de uma enfardadeira regulada para produzir fardos com $15 \mathrm{~kg}$ aproximadamente. A produção média de palha foi de $2880 \mathrm{~kg} / \mathrm{ha}$. Antecedendo à alimentação, a palha foi triturada com um triturador que desestruturou, principalmente, os colmos, resultando fragmentos inferiores a quatro centímetros. 
Nos quatro tratamentos, a dieta com $12 \%$ de PB continha na MS oferecida uma relação volumoso:concentrado de 67,5:32,5. A fração concentrada foi composta por farelo de soja, milho grão moído, uréia, cloreto de sódio e farinha de osso autoclavada; a composição percentual da dieta é apresentada na tabela 1. Os animais foram adaptados às dietas e manejo alimentar durante 14 dias, passando posteriormente para o período experimental que teve uma duração de 112 dias. Utilizou-se um confinamento parcialmente coberto com oito piquetes, sendo cada um provido de um comedouro de madeira e um bebedouro de concreto com nível regulado por torneira automática, para cada dois piquetes. Os animais foram alimentados ad libitum duas vezes ao dia, sendo a metade da dieta fornecida às 8 horas e o restante às 16 horas. A oferta de alimento foi $10 \%$ superior ao consumo voluntário diário. Diariamente, pela manhã, antes da alimentação, eram retiradas as sobras do dia anterior e pesadas para ajustes de consumo e posterior cálculo de consumo e conversão alimentar.

Foram utilizados 24 novilhos da raça Charolês, com peso médio inicial de $265 \pm 1,65 \mathrm{~kg}$ e idade média inicial de 18 meses. Os animais foram pesados no início e final do período experimental, com pesagens intermediárias a cada 28 dias, sempre obedecendo a um período de jejum de sólidos de 12 horas. Durante o período de adaptação, foram coletadas amostras da silagem de milho, de palha de trevo vesiculoso, de farelo de soja e de milho em grão para análise de matéria seca (MS) e de proteína bruta (PB). Durante o período experimental, foram coletadas amostras desses ingredientes a cada duas semanas para posterior análise bromatológica. A partir dessas amostras, foram realizadas análises de proteína bruta $(\mathrm{PB})$, fibra em detergente neutro

Tabela 1 - Composição percentual da matéria seca da dieta oferecida durante o período experimental.

\begin{tabular}{|c|c|c|c|c|}
\hline \multirow[t]{2}{*}{ Alimento } & \multicolumn{4}{|c|}{ Tratamentos } \\
\hline & T0 & $\mathrm{T} 25$ & T50 & $\mathrm{T} 75$ \\
\hline Silagem de milho & 67,5 & 50,6 & 33,75 & 16,9 \\
\hline Palha de trevo vesiculoso & 0 & 16,9 & 33,75 & 50,6 \\
\hline Grão de milho triturado & 21,1 & 23,1 & 23,5 & 24,2 \\
\hline Farelo de soja & 9,9 & 7,8 & 8,0 & 7,3 \\
\hline Uréia & 0,6 & 0,8 & 0,7 & 0,7 \\
\hline Farinha de osso & 0,6 & 0,5 & 0 & 0 \\
\hline Sal comum & 0,3 & 0,3 & 0,3 & 0,3 \\
\hline
\end{tabular}

Energia digestível da dieta, Mcal/kg MS 2,854 2,627 2,413 2,189
(FDN) e ácido (FDA), digestibilidade in vitro da matéria seca (DIVMS) e da matéria orgânica (DIVMO). Energia digestivel (ED) e o NDT foram estimados pela fórmula predita pelo ARC (1980).

O delineamento experimental foi o inteiramente casualizado com duas repetições, cada uma com três animais. Os dados foram submetidos à análise de variância e regressão através do programa estatístico SAS (1993). A análise de cada variável seguiu o modelo matemático: $\boldsymbol{Y}_{i j}=\boldsymbol{\mu}+\boldsymbol{T}_{\boldsymbol{i}}+\boldsymbol{e}_{i j}$, em que: $\boldsymbol{Y}_{i j}=$ variável dependente; $\boldsymbol{\mu}=$ média geral; $\boldsymbol{T}_{\boldsymbol{i}}=$ efeito do tratamento de índice $i ; \boldsymbol{e}_{i j}=$ erro aleatório associado a cada observação.

\section{RESULTADOS E DISCUSSÃO}

Os resultados da análise bromatológica dos alimentos, utilizados na composição da dieta fornecida aos animais, são apresentados na tabela 2. Observa-se que a palha de trevo vesiculoso apresentou um elevado teor protéico $(7,14 \%)$ quando comparado com outros tipos de palha, como a palha de aveia que apresenta, segundo FLOSS et al. (1981), 3,6\% de PB e a palha de soja cujo teor de PB é estimado por RESTLE $\boldsymbol{e}$ t al. (2000) em 3,5\%. O teor de FDN $(77,55 \%)$ da palha de trevo vesiculoso foi inferior ao verificado por QUEIROZ et al. (1998) para palha de milho $(80,16 \%)$ e por RESTLE $\boldsymbol{e t}$ al. (2000) para palha de soja $(79,1 \%)$. Em relação ao teor de FDA $(53,34 \%)$, a palha apresentou menor teor quando comparado com os valores encontrados por CRUZ (1992) para palha de soja $(64,1 \%)$ e por RESTLE $\boldsymbol{e t}$ al. (2000) também com palha de soja $(59,4 \%)$. No entanto, o teor de FDA da palha de trevo foi superior ao valor encontrado por QUEIROZ et al. (1998) para palha de milho $(51,98 \%)$. A digestibilidade in vitro da matéria seca (DIVMS) e da matéria orgânica (DIVMO) foi de 39,55 e $29,98 \%$, respectivamente. A concentração de energia digestível $(1,35 \mathrm{Mcal} / \mathrm{kg} \mathrm{MS})$ e o percentual de NDT $(30,95 \%)$ da palha de trevo vesiculoso foi inferior aos valores verificados por RESTLE $\boldsymbol{e t}$ al. (2000) com palha de soja, sendo de 1,64Mcal/kg MS e de $37,3 \%$, respectivamente.

As duas formas de expressar o consumo voluntário de matéria seca (CMS) foram afetadas, significativamente, pelo nível de palha de trevo vesiculoso na fração volumosa da dieta. $\mathrm{O}$ consumo de matéria seca expresso em $\mathrm{g} / \mathrm{kg} \quad \mathrm{PV}^{0,75}$ (CMSUTM) e em kg de MS/100kg PV (CMSP) decresceu linearmente. O CMSUTM seguiu a equação de regressão 102,9037 - 0,0765 NS ( $\mathrm{P}<0,0033$; $\left.\mathrm{R}^{2}=0,78\right)$ e o CMSP seguiu a equação de regressão apresentada na figura 1 . Pode-se verificar que, para cada unidade percentual de substituição de silagem 
Tabela 2 - Matéria seca (MS), proteína bruta (PB), fibra em detergente neutro (FDN) e ácido (FDA), digestibilidade in vitro da MS (DIVMS) e da matéria orgânica (DIVMO), energia digestível (ED) e nutrientes digestíveis totais (NDT) dos alimentos utilizados na formulação da dieta fornecida aos animais.

\begin{tabular}{|c|c|c|c|c|c|c|c|c|}
\hline \multirow[t]{2}{*}{ Alimentos } & \multirow[t]{2}{*}{ MS } & \multirow[t]{2}{*}{ PB } & \multirow{2}{*}{$\begin{array}{l}\text { FDN } \\
\%-----\end{array}$} & \multirow{2}{*}{ FDA } & \multicolumn{2}{|c|}{ Dig. In vitro, \% } & \multirow{2}{*}{$\begin{array}{c}\text { ED } \\
\text { Mcal/kg MS }\end{array}$} & \multirow{2}{*}{$\begin{array}{l}\text { NDT } \\
(\%)\end{array}$} \\
\hline & & & & & MS & MO & & \\
\hline Palha trevo vesiculoso & 87,7 & 7,14 & 77,55 & 53,34 & 39,55 & 31,84 & 1,362 & 30,95 \\
\hline Silagem de milho & 35,8 & 6,33 & 55,23 & 33,11 & 66,56 & 63,14 & 2,720 & 61,81 \\
\hline Milho & 87,1 & 9,78 & - & - & 81,76 & 77,33 & 3,382 & 76,91 \\
\hline Farelo de soja & 87,4 & 41,03 & - & - & 83,21 & 72,67 & 3,067 & 70,66 \\
\hline
\end{tabular}

palha de trevo apresentou 1,362Mcal de ED/kg de MS e a silagem de milho 2,720Mcal de $\mathrm{ED} / \mathrm{kg}$ de MS. Verifica-se na tabela 1 que o aumento de palha causou uma redução na concentração energética da dieta, sendo o teor de energia digestível de 2,854; 2,627; 2,413 e $2,189 \mathrm{Mcal} / \mathrm{kg} \mathrm{MS}$, respectivamente, para T0, T25, T50 e T75. O CMSP, citado na mesma ordem, foi 2,$49 ; 2,45 ; 2,44$ e $2,38 \%$. Observa-se que, percentualmente, a queda foi maior na concentração da energia digestível da dieta do que no

de milho por palha de trevo vesiculoso na fração volumosa da dieta, houve um decréscimo no consumo de matéria seca de $0,076 \mathrm{~g} / \mathrm{kg} \mathrm{PV}^{0,75}$ e de $0,001 \mathrm{~kg}$ por $100 \mathrm{~kg}$ de peso vivo. Segundo MERTENS (1992), o consumo de alimento é regulado por vários fatores referentes: ao animal (peso vivo, nível de produção, estado fisiológico, etc.), ao alimento (fibra, demanda energética, volume, etc.) e às condições de alimentação (disponibilidade de alimento, freqüência de alimentação, tempo de acesso ao alimento, etc.). Além disso, fatores ambientais aos quais o animal está exposto também podem afetar o consumo (NRC, 1996). A redução no CMS, à medida que aumentou o teor de palha de trevo na fração volumosa da dieta, foi o elevado teor de fibra e a baixa digestibilidade da palha de trevo (Tabela 2). Segundo VAN SOEST (1982), é através da taxa de digestão e da taxa de passagem do alimento que o conteúdo do rúmen é aliviado. MERTENS (1992) relata que em dietas com baixa densidade energética (teor de fibra elevado) em relação ao requerimento do animal, o consumo será limitado pelo efeito do enchimento do alimento.

Conforme pode ser verificado na tabela 1 , com o aumento da palha de trevo vesiculoso, caiu proporcionalmente a concentração energética da dieta. Essa redução foi causada pelo aumento do teor de FDN à medida que ocorreu maior participação de palha de trevo. A equação de regressão para consumo médio diário de energia digestível (CED) = 24,28787 - 0,09791 NS ( $\left.\mathrm{P}<0,0001 ; \mathrm{R}^{2}=0,96\right)$ mostra que o CED decresceu $0,097 \mathrm{Mcal} /$ dia para cada unidade percentual de substituição de silagem de milho por palha de trevo vesiculoso na fração volumosa da dieta. A redução no CED/animal/dia foi consequiência da queda no CMS e da redução da concentração de energia da dieta, visto que a palha de trevo vesiculoso apresentou $50 \%$ menos energia digestível que a silagem de milho. Conforme mostra a tabela 2 , a consumo de matéria seca. Relação semelhante também foi verificada por RESTLE et al. (2000) com terneiros confinados e alimentados com 0,33 e $66 \%$ de palha de soja na fração volumosa da dieta. Os autores citam que o CMS caiu em 9,5 e $13,6 \%$ ao passo que o CED caiu em 14,8 e $23,9 \%$ quando o teor de palha de soja na fração volumosa da dieta aumentou de zero para 33 e para 66\%. Segundo OSPINA \& PRATES (1998), com animais de altas exigências nutricionais alimentados exclusivamente com feno de qualidade média, os fatores relacionados ao consumo foram mais relevantes que os fatores relacionados à digestibilidade na determinação do consumo de nutrientes digestíveis, o que não foi verificado no presente experimento.

O consumo de energia digestível expresso por unidade de peso metabólico (CED Mcal $/ \mathrm{kg}$ $\mathrm{PV}^{0,75}$ ) seguiu a equação de regressão (CEDUTM) = $0,300781-0,0011 \mathrm{NS}\left(\mathrm{P}<0,0001 ; \mathrm{R}^{2}=0,96\right)$, na qual se pode observar que o CEDUTM decresce 0,0011

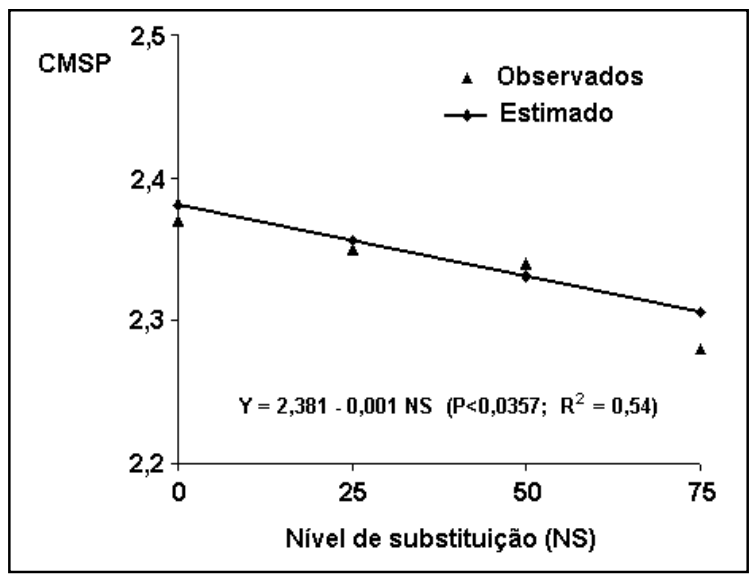

Figura 1 - Relação entre consumo de matéria seca por $100 \mathrm{~kg}$ peso vivo (CMSP), e nível de substituição de palha de trevo vesiculoso na fração volumosa da dieta. 
$\mathrm{Mcal} / \mathrm{kg} \mathrm{PV}^{0,75}$ para cada unidade percentual de substituição de silagem de milho por palha de trevo vesiculoso na fração volumosa da dieta. Com relação ao CED expresso por $100 \mathrm{~kg}$ de peso vivo, a figura 2 mostra que para cada unidade percentual de substituição de silagem de milho por palha de trevo vesiculoso na fração volumosa da dieta ocorreu uma queda de 0,0259Mcal/100 $\mathrm{kg}$ PV. RESTLE et al. (2000), ao traçarem equações para estimar o CED por $100 \mathrm{~kg}$ PV e $\mathrm{PV}^{0,75}$, verificaram que o CED decresceu em $0,0127 \mathrm{Mcal} / \mathrm{dia} \quad \mathrm{e}$ 0,0007Mcal/dia, respectivamente, para cada unidade percentual de substituição da silagem de sorgo por palha de soja.

$\mathrm{Na}$ tabela 3, verifica-se que as médias observadas para ganho de peso médio diário foram de 1,$25 ; 1,18 ; 1,07$ e 1,01kg/animal, respectivamente. Como mostra a equação de regressão apresentada na Figura 3, o ganho de peso médio diário dos animais decresceu $3,47 \mathrm{~g}$ para cada unidade percentual de substituição de silagem de milho por palha de trevo vesiculoso na fração volumosa da dieta. A queda no GMD foi uma consequiência da redução do CED. Conforme pode ser observado na tabela 3, o CEDP foi de 7,$22 ; 6,57 ; 5,98$ e $5,25 \mathrm{Mcal} / 100 \mathrm{~kg}$ peso vivo, respectivamente, para T0, T25, T50 e T75. Verificou-se que a queda de energia percentual consumida do tratamento sem palha para o tratamento com $75 \%$ de palha de trevo vesiculoso, na fração volumosa da dieta, foi de 1,97Mcal/dia, o que resultou em uma redução de $240 \mathrm{~g}$ no ganho de peso médio diário.

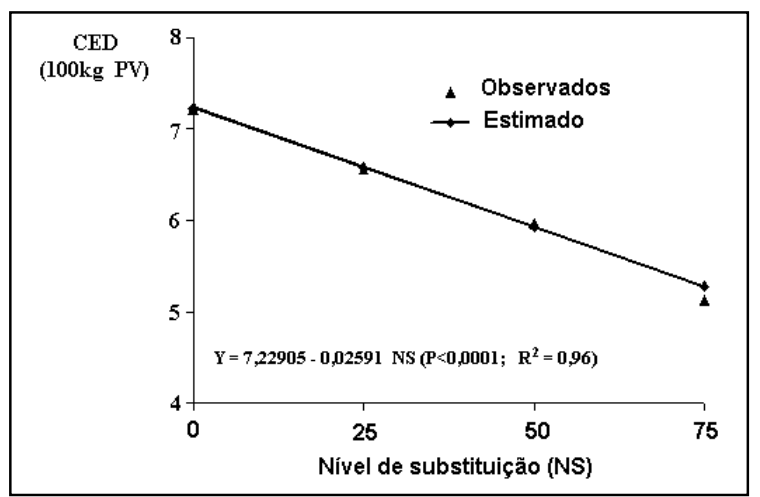

Figura 2 - Relação entre consumo de energia digestível (CED) por $100 \mathrm{~kg}$ PV e nível de substituição de palha de trevo vesiculoso na fração volumosa da dieta.
Apesar da inclusão da palha de trevo vesiculoso causar uma queda no ganho de peso, deve ser considerado que o custo de produção da silagem é bem superior ao da palha de trevo. Ocorre que a palha é um subproduto da produção de sementes, sendo que os custos para sua utilização referem-se ao processo de enfardar, o barbante utilizado e o recolhimento dos fardos. A colheita das semente já deixa o material pronto para ser enfardado sem nenhum custo adicional, resultando também em pouca mão-deobra necessária no processo de utilização da palha.

A queda no ganho de peso dos animais já é esperada quando se utiliza palhas na sua alimentação (RESTLE $\boldsymbol{e t}$ al. 2000). No entanto, o importante é quantificar essas reduções. Quedas no GMD também foram verificadas por PACOLA et al. (1983), utilizando ponta de cana-de-açúcar mais palha de soja na engorda de novilhos em confinamento, o GMD foi de 0,$611 ; 0,459 ; 0,301$ e $0,098 \mathrm{~kg}$ com o aumento de palha de soja de 70, 80, 90 para $100 \%$ de substituição na fração volumosa da dieta, respectivamente. CRUZ (1992), utilizando restos de culturas ou palhas para novilhos em crescimento, verificou que o GMD decresceu com o aumento da palha de soja na dieta, sendo de $1,32 \mathrm{~kg}$ quando a proporção de palha foi de $15 \%$, e caiu para $0,91 \mathrm{~kg}$ quando a proporção de palha foi de $40 \%$ da MS. O mesmo autor verificou que o GMD decresceu de $1,28 \mathrm{~kg}$ para $0,81 \mathrm{~kg}$ quando a proporção de palha de milho na MS da dieta aumentou de 10 para 50\%. Já RESTLE $\boldsymbol{e t}$ al. (1992) verificaram um GMD de $0,505 \mathrm{~kg}$, que foi bem inferior ao do presente experimento, e obtido com terneiros desmamados aos 90 dias, alimentados com uma dieta com $70 \%$ de volumoso constituído por $60 \%$ de palha de trevo vesicu- 


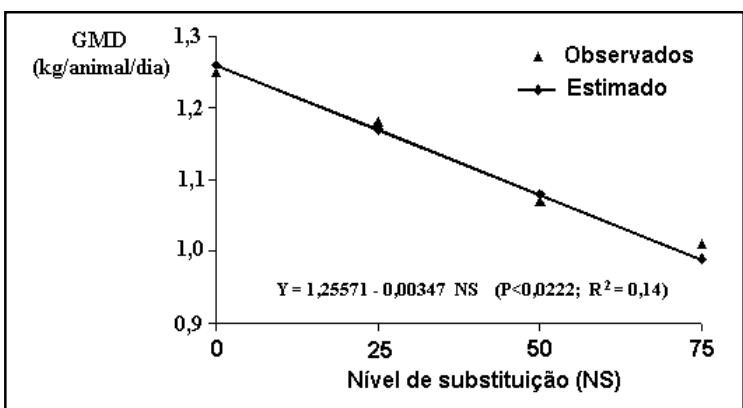

Figura 3 - Relação entre ganho de peso médio diário (GMD) e nível de substituição de palha de trevo vesiculoso na fração volumosa da dieta.

loso e $40 \%$ de silagem de sorgo forrageiro. O ganho de peso dos animais do T0 foi similar ao GMD de $1,21 \mathrm{~kg}$, verificado por RESTLE et al. (1995) com novilhos Charolês de dois anos terminados em confinamento e alimentados com $45 \%$ de concentrado mais $55 \%$ de volumoso $(50 \%$ de silagem de milho mais $50 \%$ de cana-de-açúcar picada). Enquanto que o GMD do T75 foi bem superior aos $0,713 \mathrm{~kg}$ relatado por MOLETTA \& RESTLE (1992) e obtido com novilhos Charolês de dois anos, terminados em confinamento e alimentados com cana-de-açúcar picada $(72,24 \%)$ mais concentrado $(27,76 \%)$ e que consumiram 16,05Mcal de ED/animal/dia.

A conversão alimentar (CA) entendida como a quantidade de matéria seca consumida para produzir um quilograma de ganho de peso foi afetada pelo nível de palha de trevo vesiculoso na dieta. Conforme a equação de regressão $\mathrm{Y}=6,505+0,017$ NS $\left(\mathrm{P}<0,0329 ; \mathrm{R}^{2}=0,55\right)$, a conversão alimentar piorou em 0,017 para cada unidade percentual de substituição de silagem de milho por palha de trevo vesiculoso na fração volumosa da dieta. Aumento semelhante na conversão da matéria seca consumida em ganho de peso também foi verificado por RESTLE et al. (2000), utilizando palha de soja em substituição à silagem de sorgo forrageiro, sendo o valor de 0,023 para cada aumento percentual de substituição. Os autores atribuem a queda na eficiência à redução do CMS e da concentração energética da dieta, o que causa uma redução no consumo de energia digestível, o que também foi observado nesse experimento. A eficiência na transformação de energia digestível consumida em ganho de peso também foi afetada $(\mathrm{P}<0,0284)$ pelo nível de palha na dieta, conforme a equação de regressão $\mathrm{Y}=$ 19,438475 - 0,03379 NS. A eficiência energética piorou em 0,033 para cada unidade percentual de substituição de silagem de milho por palha de trevo vesiculoso na fração volumosa da dieta.
O desempenho dos animais no presente experimento, mesmo no nível mais alto de substituição (T75), foi levemente superior a um quilograma, o que pode ser considerado satisfatório, em nível prático em confinamento. Esses resultados indicam que as palhas obtidas na produção de grãos ou sementes são uma alternativa viável na produção de bovinos e cujo potencial precisa ser melhor aproveitado no nosso meio. A utilização de subprodutos como as palhas, cujo custo de elaboração é baixo, podem tornar o sistema de produção primária mais eficiente e sustentável.

\section{CONCLUSÕES}

A substituição gradual da silagem de milho pela palha de trevo vesiculoso causa decréscimo linear no consumo de matéria seca e energia digestível, no ganho de peso médio diário e na eficiência alimentar e energética. No entanto, apesar de causar uma redução no desempenho animal, a inclusão de palha de trevo vesiculoso na fração volumosa da dieta de bovinos é viável, tendo em vista que, mesmo no nível de substituição de $75 \%$, o ganho médio diário dos animais é de um quilograma.

\section{REFERÊNCIAS BIBLIOGRÁFICAS}

A.R.C. Agricultural Research Council. The nutrient requirements of ruminant livestock. Surrey : The Gressham, 1980. 351p.

ANDERSON, D.C. Use of cereal residues in beef cattle productions systems. Journal of Animal Science. v.46, n.3, p.849-861. 1978.

BAGLEY, C.P., MORRISON, D.G., FEAZEL, J.I., et. al. Influence of roughage on wintering beef heifer performance. Nutrition Reports International, v.39, n.3, p.575-585, 1989.

CRUZ, G.M. da. Utilização dos restos de culturas e palhas na alimentação de ruminantes. In: SIMPÓSIO DE UTILIZAÇÃO DE SUBPRODUTOS AGROINDUSTRIAIS E RESÍDUOS DE COLHEITA NA ALIMENTAÇÃO DE RUMINANTES, 1992, São Carlos. Anais... São Carlos : EMBRAPA, 1992. p.99-121.

FLOSS, E.L., ARGENTA, J.A, RODRIGUES, O., et al. Avaliação de 8 cultivares de aveia (Avena ssp.) para rendimento de forragens e grãos sob diferentes freqüências de corte. Passo Fundo : Embrapa, Unidade de Passo Fundo RS, 1981. 110p. Boletim de Pesquisa 4.

MERTENS, D.R. Análise da fibra e sua utilização na avaliação de alimentos e formulação de rações. In: SIMPÓSIO INTERNAIONAL DE RUMINANTES, REUNIÃO ANUAL SOCIEDADE BRASILEIRA DE ZOOTECNIA, 29, 1992, Lavras, MG. Anais... Lavras : Sociedade Brasileira de Zootecnia, 1992. p.188-219. 
MOLETTA, J.L., RESTLE, J. Desempenho em confinamento de novilhos de diferentes grupos genéticos. Ciência Rural. v.22, n.2, p.227-233, 1992 .

MÜLLER, I., RESTLE, J., STILES, D.A. Utilization of agriculture residues. I supplementation of soybean straw for growing beef calves. Journal of Animal Science, Champaign, v.49, n.1, p.269, 1979.

NRC, National Research Council. Nutrient Requerements of beef cattle. $7^{\text {th }}$ ed. Washington : National Academy, 1996. 404 p.

OSPINA, H., PRATES, Ê.R. Efeito de quatro níveis de oferta de feno sobre o consumo de nutrientes digestíveis por bezerros. Revista da Sociedade Brasileira de Zootecnia, Viçosa, v.27, n.4, p.809-914, 1998.

PACOLA, L.J., CAIELLI, E.L., MATTOS, J.C.A. de. Ponta de cana-de-açúcar queimada mais palha de soja na engorda de bovinos confinados. Boletim da Industria Animal, Nova Odessa, v.40, n.2, p.195-199, jul./dez. 1983.

QUADROS, F.L.V. de; MARASCHIN, G.E. Desempenho animal em misturas de espécies forrageiras de estação fria. Pesquisa Agropecuária Brasileira, Brasília, v.22, n.5, p.535-541, 1987.

QUEIROZ, A.C. de; BARBOSA, M.A., RESENDE, F.D. de; $\boldsymbol{e}$ al. Suplementação da palhada de milho na alimentação de bovinos. 1. Consumo, taxa de passagem da matéria seca, degradação in situ da matéria seca e da fibra em detergente neutro. Revista da Sociedade Brasileira de Zootecnia,
Viçosa, v.27, n.2, p.381-389, 1998.

RESTLE, J., ALMEIDA, S.R.S. de; OURIQUE, N. L. Comparação de diferentes tipos de fenos na alimentação de terneiros de corte. In: REUNIÃO ANUAL SOCIEDADE BRASILEIRA DE ZOOTECNIA, 1992, Lavras, MG. Anais... Lavras : Sociedade Brasileira Zootecnia, 1992. p.143.

RESTle, J., ALVES FILHO, D.C., BRONDANI, I.L., $\boldsymbol{e}$ t al. Palha de soja (Glicyne max) como substituto parcial da silagem de sorgo forrageiro (Sorghum bicolor (L.) Moench) na alimentação de terneiros de corte confinados. Ciência Rural, Santa Maria, v.30, n.2, p.311-316, 2000.

RESTLE, J., FELTEN, G.H., VAZ, F.N. Efeito de raça e heterose para desempenho em confinamento de novilhos de corte. In: REUNION LATINOAMERICANA DE PRODUCCION ANIMAL, XIV, Argentina, Revista Argentina de Produccion Animal. v.15, n.3-4, p.852-856, 1995.

SAS INSTITUTE, SAS/STAT. User's Guide: Statistics, 4, Version 6. Cary, NC, USA : SAS Institute, 1993. v.3. 943p.

SILVA, J.L.S. da, SAIBRO, J.C. de, FREITAS, F.R. de, $\boldsymbol{e}$ t al. Produtividade animal em diferentes pastagens de inverno em planossolo no litoral norte no RS. In: REUNIÃO ANUAL DA SOCIEDADE BRASILEIRA DE ZOOTECNIA, 34, 1997, Juiz de Fora - MG. Anais... Juiz de Fora : SBZ, 1997. p.279-281p.

VAN SOEST. Nutritional ecology of the ruminant. Corvallis : O \& Books, 1982. 374p.

Ciência Rural, v. 30, n. 2, 2000. 